\title{
Experimental and Numerical Investigation of Metal Type and Thickness Effects on the Impact Resistance of Fiber Metal Laminates
}

\author{
M. Sadighi • T. Pärnänen • R. C. Alderliesten • \\ M. Sayeaftabi $\cdot$ R. Benedictus
}

Received: 1 August 2011 / Accepted: 14 October 2011 /Published online: 27 October 2011

(C) Springer Science+Business Media B.V. 2011

\begin{abstract}
The impact response of fiber metal laminates (FMLs), has been investigated with experiments and numerical simulations, which is reported in this article. Low-velocity impacts were carried out to study the effects of metal type and thickness within FMLs. Glare5-3/2 laminates with two aluminum layer thicknesses and a similar FML containing magnesium sheets were impacted by drop weight tests. Also, a major part of this study was to accomplish a dynamic non-linear transient analysis to study the impact response of FMLs using the commercial finite element (FE) analysis code ABAQUS. By reviewing different approaches of modeling constituents of an FML, it is shown that the appropriate selection of elements has more significant role than failure criterion to predict acceptable results for this type of laminate and loading. The good agreement obtained between experimental and numerical results verifies the possibility of relatively simpler simulation by FE-analysis to predict overall response of FMLs under impact loading.
\end{abstract}

Keywords Fiber metal laminates · Magnesium · Low velocity impact · Numerical modeling · Abaqus

\section{Introduction}

During the last two decades, the fiber metal laminate (FML) concept has been developed to provide an answer to the need of improving material properties and reducing structural weight of primary aircraft structures. This material concept exhibits excellent fatigue and impact resistance as compared to either monolithic aluminum or carbon fiber reinforced

M. Sadighi $(\bowtie) \cdot$ M. Sayeaftabi

Mechanical Engineering Department, Amirkabir University of Technology, Tehran, Iran

e-mail: mojtaba@aut.ac.ir

T. Pärnänen

School of Science and Technology, Aalto University, Helsinki, Finland

R. C. Alderliesten $\cdot$ R. Benedictus

Faculty of Aerospace Engineering, Delft University of Technology (TUDelft), Delft, The Netherlands 
composite materials $[1,2]$. An FML for primary structures consists of thin high strength aluminum alloy sheets (typically 0.3 to $0.5 \mathrm{~mm}$ thick) bonded together with alternating unidirectional composite prepregs. Arall and Glare are the most popular FMLs where prepregs are aramid or glass fibers in an epoxy resin, respectively. A review made recently by the authors on impact resistance of FMLs showed that in spite of many articles concerned on the impact behavior of these laminates, the research on this part of FMLs' performance is still in the early stages.

In the present study, two variables were investigated experimentally and numerically for their influence on the impact performance: metal type and metal thickness. Two thicknesses for Al 2024-T3 were selected, with in addition, an FML with the aluminium substituted with $\mathrm{Mg}$ AZ31B-H24 sheets. These FMLs were tested using a drop-weight impact test. Also, FE analysis has been accomplished to model FMLs under low-velocity impact using the commercial FE analysis code ABAQUS.

The experimental results are compared with FEM predictions to show the applicability of the chosen model to predict the impact resistance of FMLs. Two approaches were selected to show the effect of element types and failure criteria on the predicted results.

\section{Background / Prior Works}

\subsection{General Findings}

Two aluminum alloys 2024-T3 and 7075-T6 (or 7475-T6), comprise the main variation used for the metal constituents of FMLs [3, 4]. Aluminum alloys of 7000 grades are stronger and more brittle whereas the 2024 alloy is more ductile and slightly stiffer. The smaller area under the stress-strain curves of Al 7075-T6 compared to Al 2024-T3 results in about 1/3 lower energy until failure for A17075-T6 [3, 4]. This property affects the impact performance of Glare, i.e. Glare1, with the 7475-T6 alloy, absorbs the energy with a fracture mode whereas more energy attenuated through local plastic deformation in Glare2 containing the more ductile Al 2024-T3. Cortes and Cantwell $[5,6]$ have listed a number of advantages of magnesium alloys over some other alloys such as low density, improved electromagnetic shielding capability and superior corrosion resistance. However, according to another study [7] the latter property seems to be rather a point of concern for magnesium. Advantages and disadvantages of magnesium based FMLs with respect to mechanical properties are investigated by Alderliesten et al. [8] to determine whether this alloy is applicable in FMLs for structural application. The only available study [6] reporting that $\mathrm{Mg}$ based FMLs offer higher specific perforation energy than the similar aluminum based FMLs, is based on data generated with a thermoplastic resin, rather than a thermoset.

In terms of metal/composite volume fraction within an FML, Fan et al. [9] reported that the difference between specific perforation energies of FMLs and plain composites decreases when the composite thickness in the FMLs increases. Vlot [10] concluded that Arall and monolithic Al 2024-T3 with a smaller thickness will have a larger restitution until first failure. This is due to the fact that the thicker material exhibits more plastic deformation at the outside of the material. A noticeable fact to justify the superior impact performance of FMLs is that thin aluminum sheets have higher specific energy absorption because of a more membrane deformation than the thicker sheets which have more dominant bending behavior. Apart from membrane deformation of the metal, the composite layers absorb energy by delamination and fiber failure. Looking at the FML as a set of separate layers, the combination of these energy absorption modes results in higher impact 
performance. Liaw et al. [11] examined the thickness effect by increasing the number of layers. They concluded that the minimum cracking energy increases following a parabolic relation as the thickness of panel increases.

Some other variables affecting impact properties of FMLs were studied by other researchers such as: type of fibers $[12,13]$, type of matrices [14-16], lay-up configuration $[4,17]$, scaling effect $[18,19]$ and geometry of impactor $[20,21]$.

\subsection{Numerical Studies}

There are a few articles in the literature [22-26] that report numerical modeling of impact resistance of FMLs. Modeling of FMLs is a challenging work due to the difficulties associated with the plastic behavior, delamination, crack growth and perforation caused by impact loading, and loading rate [27]. Reasonable FE-analysis of impact resistance of FMLs should appropriately involve the behaviour of different constituents with suitable element type selection, failure criteria and strain effects. There are two general articles [27, 28] that describe general modeling and simulation of FMLs. Laliberte et al. [22] developed a user defined material subroutine in LS-Dyna for FMLs to study damage mechanisms in Glare. They used three types of interface models: tied interface (with elastic-plastic aluminum, elastic prepregs), simple-tie break (with the same mentioned behavior as previous) and tiebreak (with elastic-plastic aluminum and damageable prepregs). It was shown that the last one provides better predictions of impact performance. Guan et al. [23] used ABAQUS/ Explicit to perform a FE simulation of the thermoplastic based FMLs subjected to impact velocities up to $150 \mathrm{~m} / \mathrm{s}$. The composite layers were modeled as an isotropic material with a tensile failure criterion and the aluminum layers were modeled as an elasto-plastic material with rate dependent behavior. They reported good agreement between resulting failure modes, maximum permanent displacement and decay of the kinetic energy of the projectile with experimental results. Song et al. [24] modeled carbon reinforced aluminum laminates (CARALL) under low velocity impact using ABAQUS software. They used shell element with Hashin's failure criterion for the composite layers and 8-node solid element without failure criterion for the aluminum alloy. In a detailed article, Seo et al. [25] presented an FE model to simulate the Glare panels with diverse impact damages. Two and three-dimensional failure criteria in the ABAQUS code were used to model stiffness degradation in the panels. For two types of incident energies, i.e. energy to create barely visible impact damage (BVID) and the energy to create clear visible impact damage (CVID), they have shown that both two and three-dimensional failure modes showed good agreement with the experimental results, having in mind that the three-dimensional model predicts the impact force and permanent displacement more realistically.

\section{Experimental Analysis}

\subsection{Selection of FML Concepts}

Two concepts were selected to examine the effect of metal type and thickness within FMLs on the impact resistance of these types of materials. The first concept is based on replacement of aluminum layers (Al 2024-T3) with magnesium sheets (Mg AZ31B-H24) with thickness of $0.5 \mathrm{~mm}$. The second concept is based on the aluminum layers thickness: $0.5 \mathrm{~mm}$ or $0.4 \mathrm{~mm}$. The layup in all specimens was according to 5-3/2 type, i.e. Glare 5-3/2 (with two thicknesses of A12024-T3) and FML with magnesium layer (Mg AZ31B-H24, 
$0.5 \mathrm{~mm}$ thickness). The composite prepreg layers in all specimens were FM94/S2 (FM94 epoxy adhesive film, S2 glass fiber, UD) with a nominal thickness after curing of $0.127 \mathrm{~mm}$. Material properties, layups and test parameters are shown in Tables 1, 2 and 3.

\subsection{Test Set-Up}

All impact tests were performed with the drop tower equipment in the structures and material laboratory of the Faculty of Aerospace Engineering of TUDelft. The drop tower consists of a carriage on two rails carrying the impactor using an electromagnet. The height of impactor before the drop is a first estimate for the impact energy to be applied. The exact data is acquired by measuring of the impact velocity right before impact. In addition, the force-time stream data measured by a strain gauge load cell within the impactor is captured. By inserting a piece of metal on the specimen after impact, a second impact as a result of bouncing is prevented. The steel impactor used in the tests has a hemisphere nose shape with a $15.9 \mathrm{~mm}$ diameter and $2760 \mathrm{~g}$ weight, while the specimens have a square dimension of 110 by $110 \mathrm{~mm}$. The specimens are clamped in a steel frame tightened by 8 steel bolts leaving an open circular area with $80 \mathrm{~mm}$ in diameter.

The setting used in the test for the force range was dependent on the pre-set height. By recording force-time history, it is possible to derive numerically force-displacement, velocitytime and energy-time curves. For every type of FML, the impact energies were varied from $10 \mathrm{~J}$ to full perforation energies.

\section{Finite Element Analysis}

\subsection{General Model}

There are 3 approaches in the literature to model FMLs under impact loading using ABAQUS /Explicit commercial FEM software. They are separated according to the type of chosen elements, mechanical behavior description and failure criteria selection for the constituents of FMLs. In the first approach [23], solid elements were used for both aluminum and composite layers. The aluminum alloy was modeled as an elasto-plastic material with rate-dependent behavior, whereas the composite was modeled as an isotropic material. Both shear and tensile failure criteria were used to simulate the failure processes in

Table 1 Material and mechanical properties of constituent sheet materials

\begin{tabular}{llll}
\hline & A1 2024-T3 & Mg AZ31B-H24 & FM94/S2 \\
\hline E [GPa] & 72.4 & 45 & $53.7\left(E_{1}\right)$ \\
& & & $9.1\left(E_{2}\right)$ \\
$\nu$ & 0.33 & 0.35 & $0.29\left(\nu_{12}\right)$ \\
$G[\mathrm{GPa}]$ & 27.2 & 16.67 & $3.4\left(G_{12}\right)$ \\
$\rho\left[\mathrm{g} / \mathrm{cm}^{3}\right]$ & 2.77 & 1.78 & 1.96 \\
$\sigma_{y}[\mathrm{MPa}]$ & 345 & 220 & - \\
$\sigma_{u l t}[\mathrm{MPa}]$ & 441 & 290 & $2277\left(\sigma_{u l t, x}\right)$ \\
& & & $49.5\left(\sigma_{u l t, y}\right)$ \\
$\varepsilon_{u l t}[\%]$ & 18 & 15 & $4.5\left(\varepsilon_{u l t, x}\right)$ \\
& & & $0.5\left(\varepsilon_{u l t, y}\right)$ \\
\hline
\end{tabular}


Table 2 Layup of the tested laminates (a Al 2024-T3 t=0,510 mm, b Al 2024-T3 t=0,404 mm, c Mg AZ31B-H24 t=0.508 mm, d FM94/S2 t=0,127 mm)

\begin{tabular}{lll}
\hline & Abbreviation & Layup \\
\hline Glare 5-3/2-0.4 & GL5-1 & $0 \mathrm{~b} / 0 \mathrm{~d} / 90 \mathrm{~d} / 90 \mathrm{~d} / 0 \mathrm{~d} / 0 \mathrm{~b} / 0 \mathrm{~d} / 90 \mathrm{~d} / 90 \mathrm{~d} / 0 \mathrm{~d} / 0 \mathrm{~b}$ \\
Glare $5-3 / 2-0.5$ & GL5-2 & $0 \mathrm{a} / 0 \mathrm{~d} / 90 \mathrm{~d} / 90 \mathrm{~d} / 0 \mathrm{~d} / 0 \mathrm{a} / 0 \mathrm{~d} / 90 \mathrm{~d} / 90 \mathrm{~d} / 0 \mathrm{~d} / 0 \mathrm{a}$ \\
Glare $5-3 / 2-0.5-\mathrm{Mg}$ & GL5-Mg & $0 \mathrm{c} / 0 \mathrm{~d} / 90 \mathrm{~d} / 90 \mathrm{~d} / 0 \mathrm{~d} / 0 \mathrm{c} / 0 \mathrm{~d} / 90 \mathrm{~d} / 90 \mathrm{~d} / 0 \mathrm{~d} / 0 \mathrm{c}$ \\
\hline
\end{tabular}

the aluminum layers and a similar tensile failure criterion used for the composite layers. The second approach [24] used 8-node solid element (C3D8R) and 8-node shell element (SC8R) for the aluminum alloy and composite layers, respectively. In the implemented model, the Hashin damage initiation criteria were used for the composite layers and no damage criterion was applied to the aluminum sheets. However, due to the fact that realistic modeling of layers and interface between the metal and fiber-reinforced polymer layers requires through-thickness stresses, shell elements with plane-stress assumption are not suitable for composite layers [25]. On the other hand, the failure criteria included in ABAQUS for composite layers are only applicable to continuum shell elements with plane stress conditions. Therefore, in the last approach, Seo et al. [25] developed a user material subroutine VUMAT to simulate progressive damage and failure of composite layers with three-dimensional solid-elements. Due to complexity and required CPU time of the third approach, the first two approaches, with some changes, are selected in the present research. Through that the relative influence of element type and failure criterion on the prediction of impact resistance of FMLs are examined.

\subsection{Model Properties}

According to the first approach [23], due to the geometrical symmetry of the specimen, only a quarter of the laminate was modeled with applying appropriate symmetry boundary conditions (Fig. 1). In Fig. 1, dark layers are made of AL 2024-T3 or Mg AZ31B-H24 and bright layers are FM94/S2. Each layer has 2 elements in thickness, 20 elements in radius direction and 10 elements in prephery. Elements chosen to the middle of the target (impact point) are smaller in radial direction since most of deformation takes place in this region. Totally each layer has 350 C3D8R elements. At the periphery of the laminate boundary (outside edge), all displacement degrees of freedom was fixed, corresponding to a perfect-clamping condition. Also, the impactor was constrained against motion in the $\mathrm{x}$ and $\mathrm{y}$ directions, or rotation about the same axes. Because the impactor was made of steel, with a larger stiffness compared with FMLs, the impactor was modeled as a perfectly rigid body. Contact between the projectile and individual

Table 3 Test parameters used in experimental and numerical parts
Test parameters

\begin{tabular}{ll}
\hline Specimen size & $110 \times 110 \mathrm{~mm}^{2}$ \\
Test area: & $\varnothing 80 \mathrm{~mm}$ (Clamped) \\
Impactor mass: & $2760 \mathrm{~g}$ \\
Impactor shape: & Hemisphere $(\varnothing 15.9 \mathrm{~mm})$ \\
Impact energy: & 20 specimens for every hybrid \\
& material with energy range $10-80 \mathrm{~J}$ \\
\hline
\end{tabular}




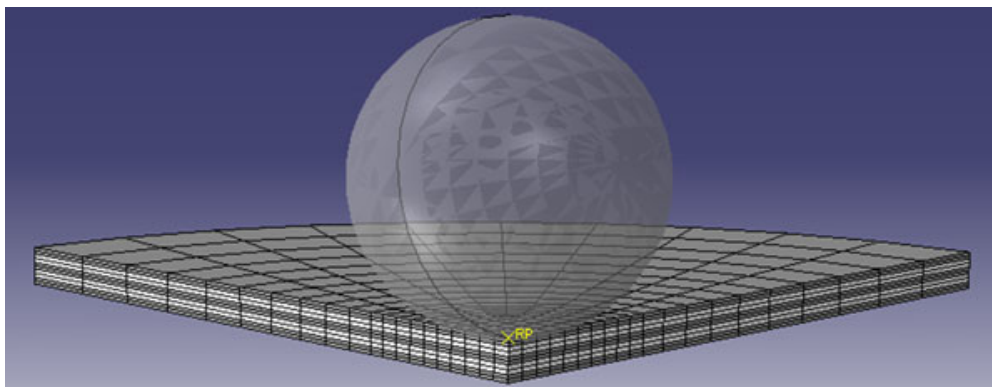

Fig. 1 Finite element model (mesh geometry). Dark layers are made of AL 2024-T3 or Mg AZ31B-H24 and bright layers are FM94/S2

laminates was modeled using a friction coefficient of 0.2 . Layers of target were tied together and had no separation.

Both shear failure and tensile failure criteria were used to simulate the failure processes of aluminum or magnesium layers. The FM94/S2 composite layer was modeled as an equivalent isotropic material, using classical laminate theory. The tensile failure criterion was used to predict failure in the FM94/S2 layers. The material properties and other conditions used for aluminum, magnesium and composite layers are given in Table 1, whereas the other conditions for the laminate and specimen are provided in Tables 2 and 3. The properties for magnesium alloy (AZ31B-H24), include rate-dependent parameters that were extracted from the curves in Fig. 2 [29].

Simulation of the failure sequence is similar as described in ref. [23]. In this way, impactor perforation can be modeled by automatically removing failed elements, which is a routine available in the ABAQUS/Explicit software package.

The second approach [24] was also adopted, which differs from the first approach in the element and failure criterion selection for composite layer; here 8-node continuum shell elements (SC8R) applying the Hashin damage initiation criteria was used. Although in ref [24], solid elements (C3D8R) were applied for aluminum alloy without any failure criterion, the present research used similar assumptions for aluminum as the first approach, i.e. elasticplastic material with rate-dependent behavior.
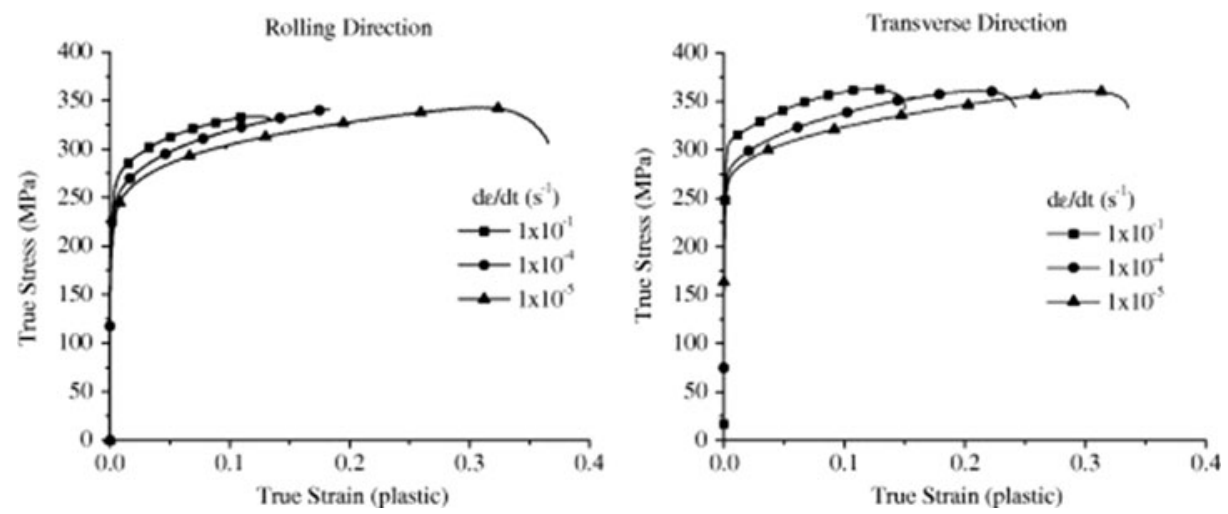

Fig. 2 Stress-strain behavior as a function of applied strain rates for rolling and transverse directions of AZ31B magnesium alloy [30] 


\section{Experimental Results}

This section presents and discusses the experimental results with respect to energy restitution coefficient, maximum impact force, maximum displacement and permanent deformation.

\subsection{Energy Restitution Coefficient (ERC)}

The Energy Restitution Coefficient (ERC) is often used to quantitively correlate impact results of different materials. Although the comparison on the basis of ERC only, has proven to be insufficient, it can be used to some extent to compare the investigated FMLs reported here. The ERC is the squared ratio of the velocity of the impactor before and after impact

$$
E R C=\frac{V_{R E B O U N D}^{2}}{V_{I M P A C T}^{2}}
$$

The squared value magnifies the difference between two data points. The value varies between zero and one, where one indicates that it is a purely elastic impact and zero means that all the energy is transferred from the impactor head to the specimen (inelastic impact). The above equations can be written in terms of absorbed and impact energies as:

$$
E R C=1-\frac{U_{\text {absorbed }}}{U_{\text {impact }}}
$$

Figure 3 shows ERC profiles against the impact energy for the three specimen types. Increasing the value of the impact energy produces a decrease in the ERC value. The zero value means that $100 \%$ of the impact energy is absorbed by the specimen. Increasing the impact energy further, results in an increase of the ERC indicating that not all the energy is absorbed, which means the specimen is perforated. Comparing ERC curves of the laminates makes clear that in the low impact energies, Mg-GL5 laminate absorbs less energy, whereas GL5-1 and GL5-2 have similar behaviour. At medium levels of impact energies ( $30 \mathrm{~J}$ to $80 \mathrm{~J}$ ), the laminates show more or less the same capacity of energy absorption, but with higher amount of damage in Mg-GL5 and GL5-1 compared to GL5-1 laminates. The highest perforation energy is observed for GL5-2 which is attributed to thicker Al layers in the FML.

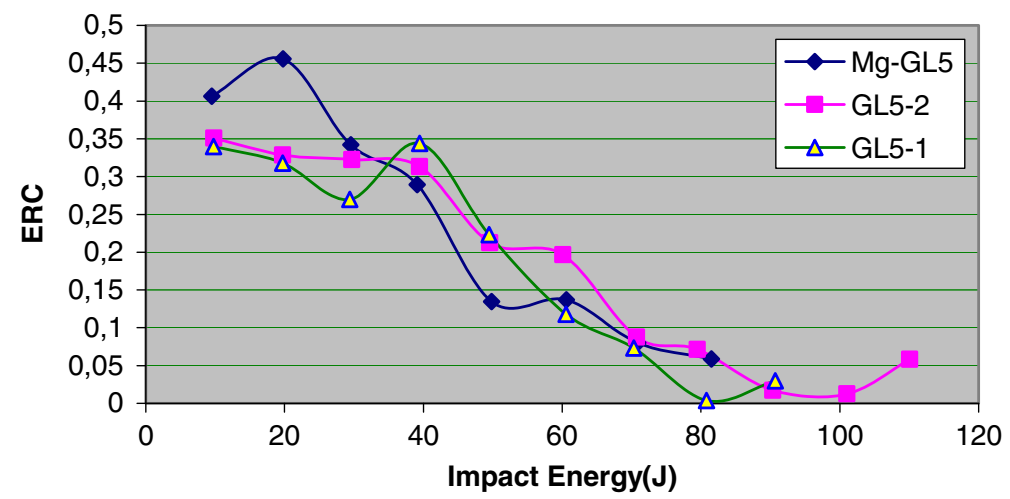

Fig. 3 ERC curves for the tested laminates 


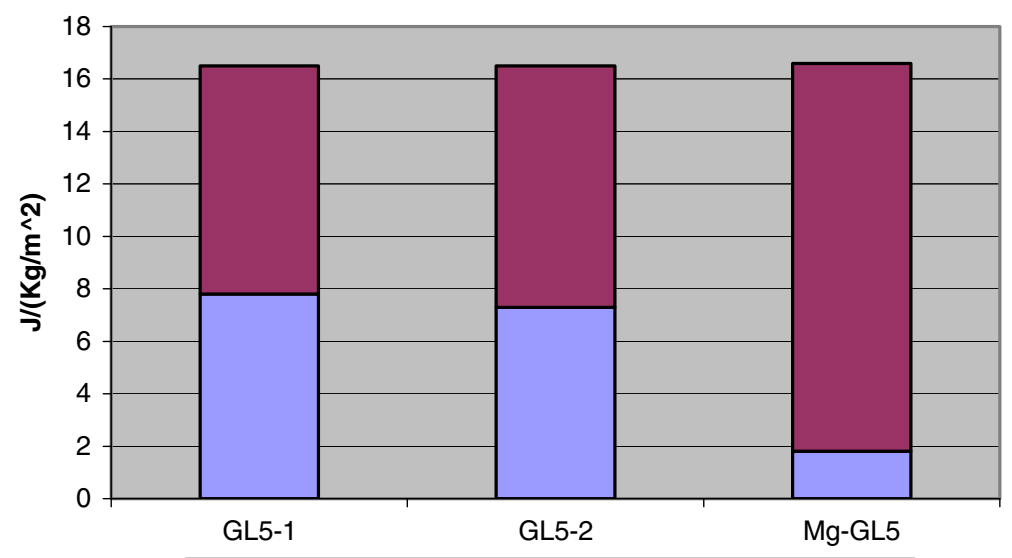

Specific Peforation Energy $\square$ Specific First Crack Energy

Fig. 4 Specific limit energies for the tested laminates

However, a reasonable criterion for a better comparison is to look at the energy to first failure and the perforation energy, both normalized by the weight of laminate. Figure 4 shows the specific crack/perforation energies (energy divided by areal density) for the three tested laminate types. It reveals that increasing the aluminum layer thickness has not improved the specific first crack energy. Also, the specific first crack energy of Mg-GL5 is less than $30 \%$ of the value for the similar laminate with aluminum layers. However, specific perforation energy of Mg-GL5 is slightly higher than GL5-2, having in mind that it is associated with a large damage area.

\subsection{Maximum Impact Force}

Figure 5 shows the maximum forces recorded during impact on the tested laminates plotted against the applied impact energy. After a steep and linear rising of the impact force with impact energy, there are plateaus in all curves, where the maximum indicates the onset of failure within the laminates. It depicts that after similar behavior at low impact energy, Mg-GL5 has the lowest magnitude of force followed by GL5-1 and GL5-2. The lower force

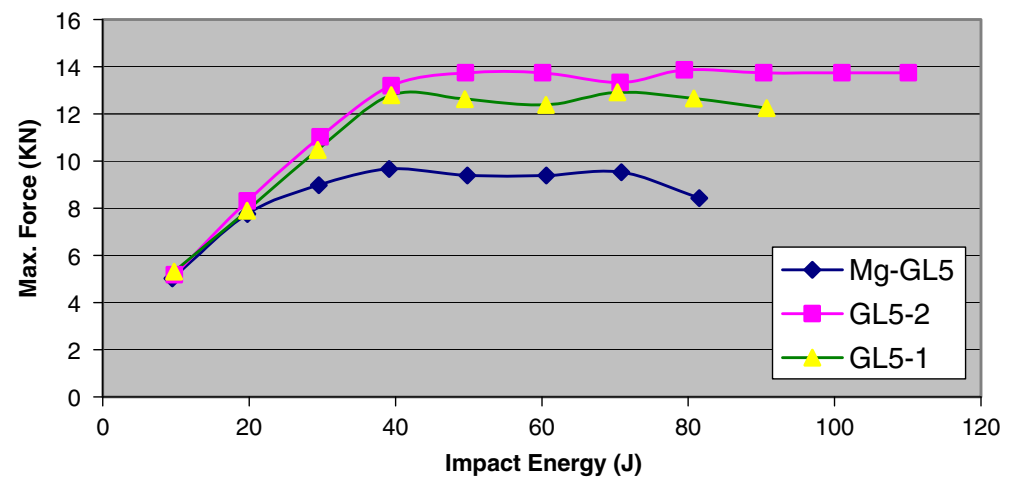

Fig. 5 Maximum impact forces for the tested laminates 


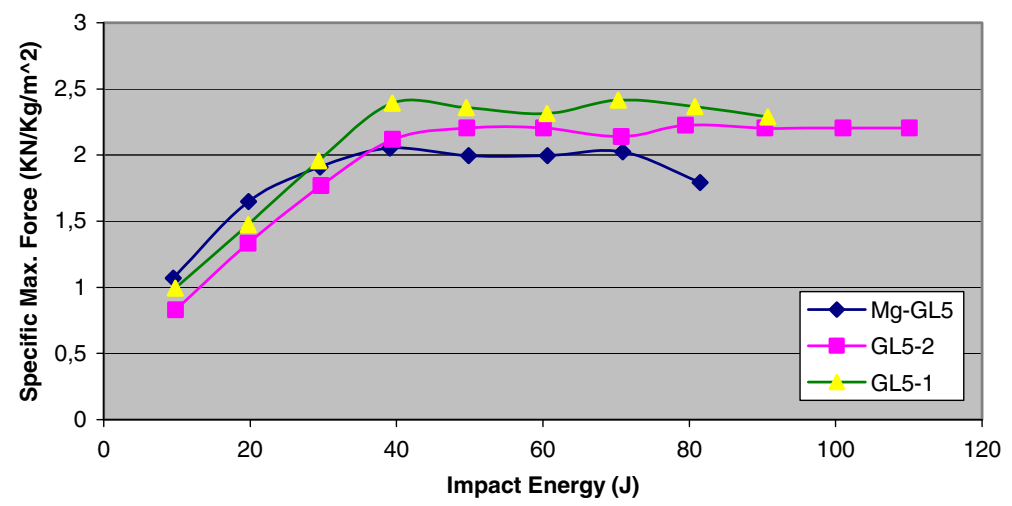

Fig. 6 Specific maximum force for the tested laminates

of Mg-GL5 is attributed to the lower modulus of elasticity of Mg layers. It can be seen that the maximum forces for GL5-1 and GL5-2 are almost the same with lower forces required to propagate damage in GL5-1. Again, normalizing maximum forces with respect to laminate weight results in the curves given in Fig. 6. The trend remains for all three curves, but first of all; all three curves are close to each other, with Mg-GL5 still the lowest, but the highest now is GL5-1 instead of GL5-2. It means that using thicker aluminum layers has not caused improvements in terms of specific maximum force.

\subsection{Maximum Displacement}

Maximum displacements of the tested laminates are shown in Fig. 7 against the impact energy. It is interesting to note that the maximum displacements caused by impact energies below $70 \mathrm{~J}$ are approximately the same for the three laminate types. The differences start when catastrophic failure (perforation associated with creating petals) is initiated within the laminates. Larger damage and fracture in the Mg-GL5 and GL5-1 accompanies with more displacement at lower impact energies comparing to GL5-2 laminates. The trends for the three curves have not changed when they are normalized by the weight of laminates.

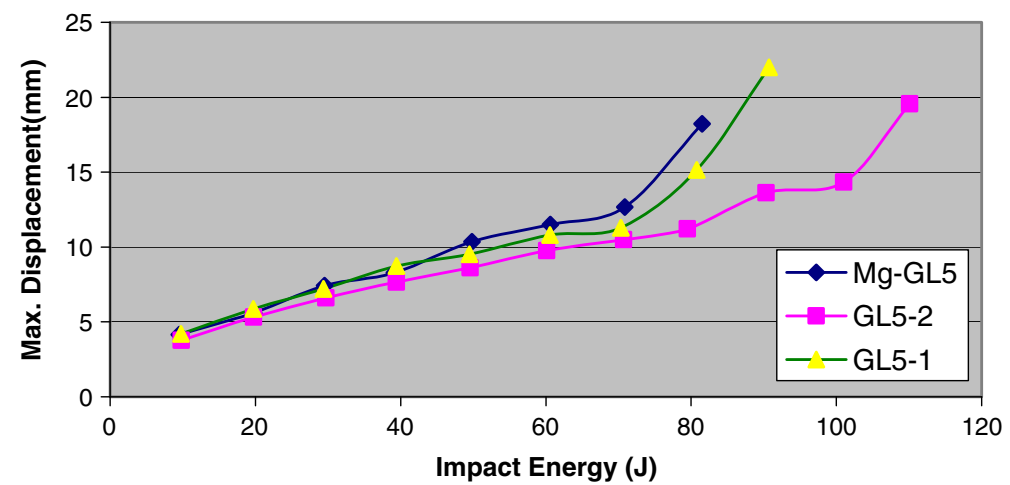

Fig. 7 Maximum displacements for the tested laminates 

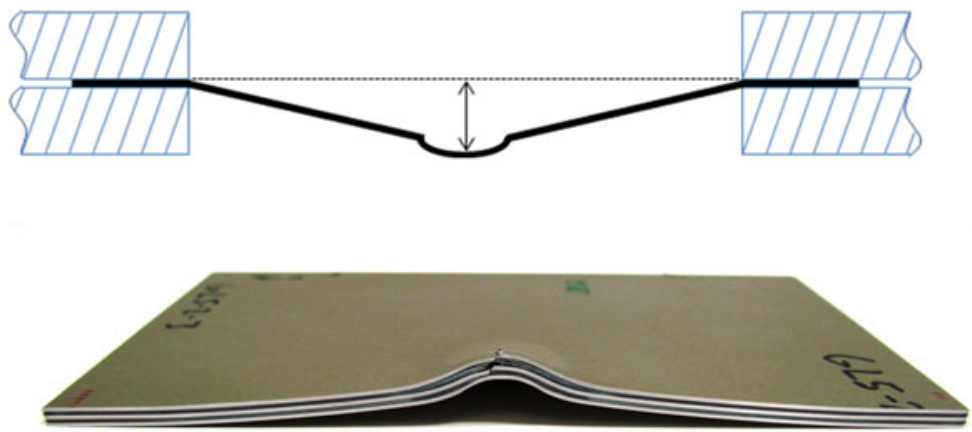

Fig. 8 The permanent deformation measurement

\subsection{Permanent Deformation}

The permanent deformation is a measurement of the center of the dent after impact which is measured while specimens were still attached in the text fixture (see Fig. 8).

The permanent deformation (or dent depth) could be more important for structural parts, such as for instance leading edges of aircraft, because a larger dent depth creates more turbulent airflow. For this reason, the magnitudes of permanent deformations are compared with each other in Fig. 9. The dent depth is influenced usually by some parameters such as the bending stiffness, the target dimensions, boundary conditions and the type of failure modes induced in the laminate. Among the three tested laminate types, Mg-GL5 shows the lowest dent depth, due to its capacity of more global deformation and less residual displacement. However, the higher dent depth of GL5-1 seems to be due to its type of failure inside the laminate. This phenomenon changes if a higher thickness of aluminum layer applied, since the resulting higher stiffness in that case leads to a deeper dent depth. Figure 10 shows specific permanent deformation (permanent deformation divided by weight). The curve for GL5-1 remains the highest, but the order between Mg-GL5 and GL5-2 has changed. Therefore, in terms of dent depth, the substitution of magnesium layers instead of aluminum layers has not lead to an improvement.

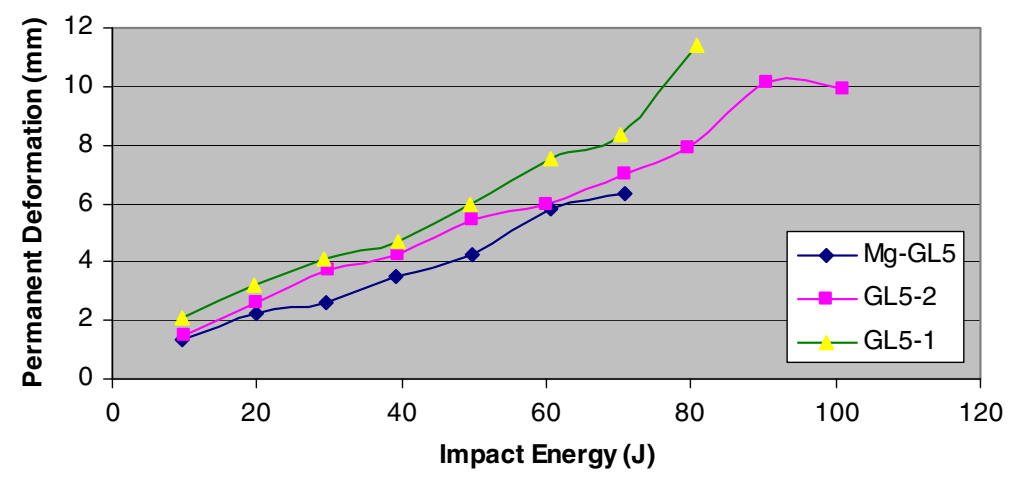

Fig. 9 Permanent deformations (maximum dent) for the tested laminates 


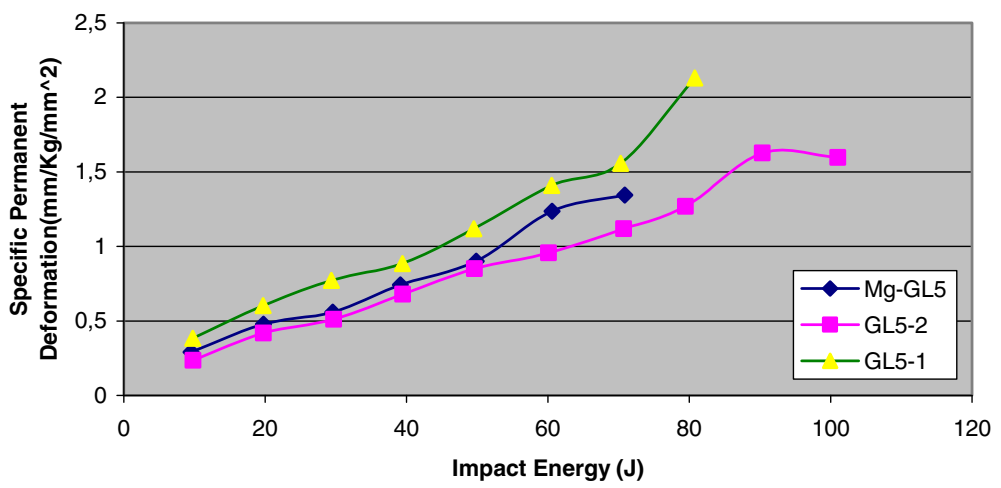

Fig. 10 Specific permanent deformations for the tested laminates

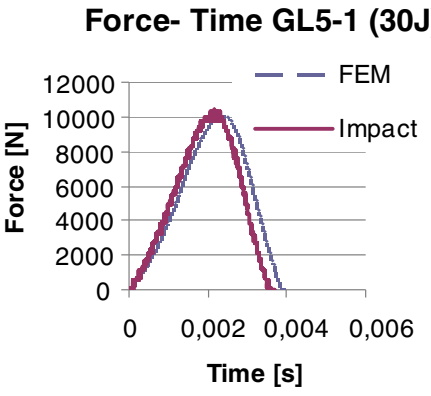

Force -Time GL5-2 (30J)

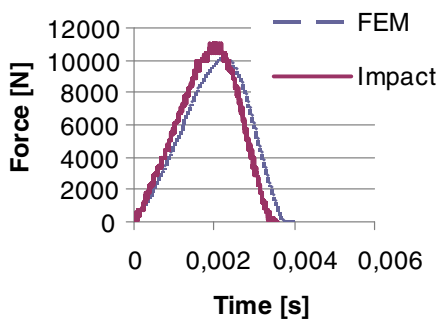

Force - Time MgGL5 (30J)

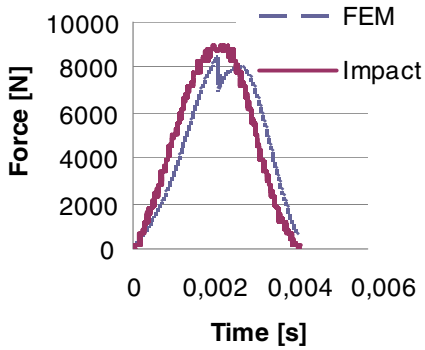

Force - Time GL5-1 (80J)

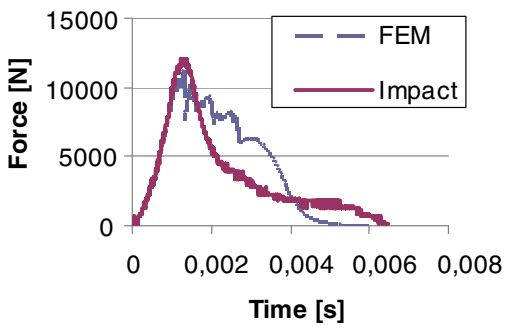

Force - Time GL5-2 (80J)

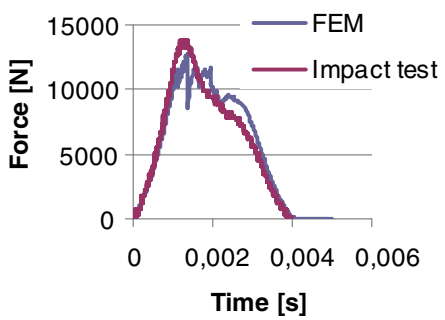

Force - Time MgGL5 ( 80J)

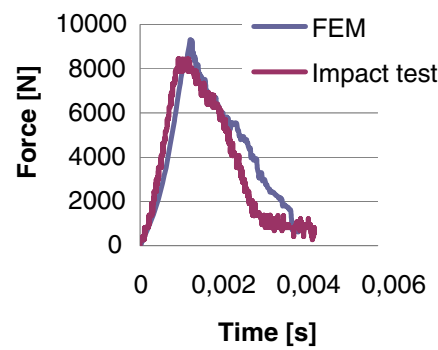

Fig. 11 Force-time histories, FEM and experimental results 


\section{Numerical Results}

\subsection{Validations}

The first approach discussed in the section 3, has been used to predict the impact resistance of the laminates considered in the present research. The predictions were subsequently compared with the experimental results. Figures 11 and 12 show this comparison for load-time history and load-displacement of the laminates, respectively. Similar curves can be obtained for velocity-time and energy-time.

There is an evident agreement between FE-predictions and experimental results, especially before taking place of catastrophic failure in the laminates. It could be expected that the applied FE-model is not capable to predict the experimental results up to final failure very well. The discrepancies between FE-predictions and experimental results are more evident for the Mg-GL5 laminates, since great amount of fracture occured within the laminates at relatively high impact energy. However, it is shown that the FE-model, which requires less computer time compared to the complex model (third approach [24]), is capable to predict overall behavior of FMLs under impact loading with sufficient accuracy.

Force - Displacement GL5-1 (30J)

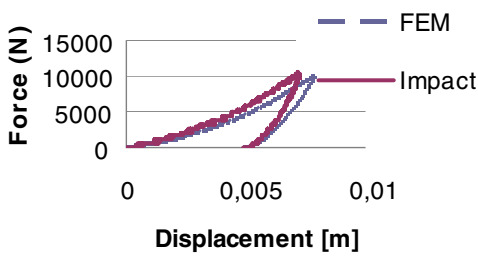

Force - Displacement GL5-2 (30J)

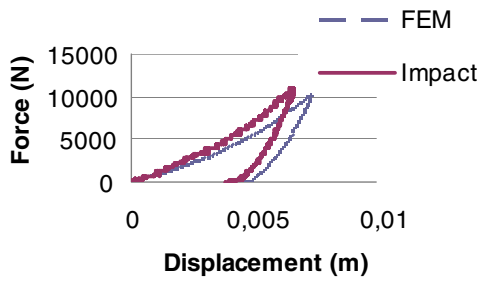

Force - Displacement MgGL5 (30J)

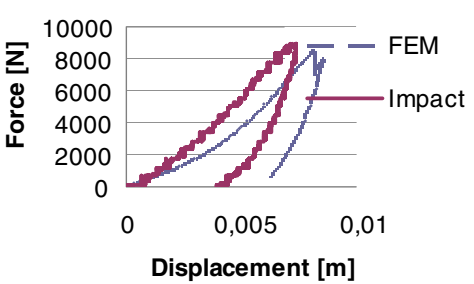

Force - Displacement GL5-1

(80J)

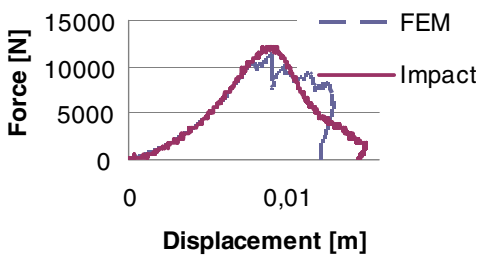

Force - Displacement GL5-2

(80J)

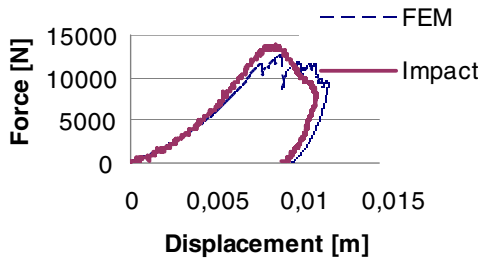

Force - Displacement MgGL5

(80J)

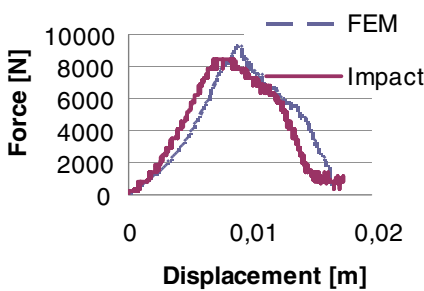

Fig. 12 Force-displacement curves, FEM and experimental results 


\subsection{Type of Element Effect}

As mentioned earlier, one of the objectives of the numerical modeling analysis in the present research is to check the applicability of the element types for composite layers within FMLs. The first approach uses solid element, while the second approach applies continuum shell element for composite layers. The failure criterion in the first approach is based on tensile strength, whereas in the second approach, Hashin's failure initiation has been used.

Figure 13 shows the results obtained by the two approaches for the GL5-1 laminate, using the properties given in Tables 1, 2 and 3. It is clear that both approaches predict similar magnitudes in good agreement with the experimental results at the initial phases of impact process, with better correlation for the first approach (solid elements). At higher levels of impact energy, the first approach is still predicting sufficient accurate, while the second approach (shell elements) becomes highly inaccurate; predicting even wrong trends. However, at high level of impact energies, this approach is not able to predict the observed behavior in FMLs. It was stated that the second approach uses continuum shell elements and Hashin failure criterion for composite layers, whereas the first approach applies solid elements and maximum tensile strength criterion. As Hashin failure criterion is an appropriate criterion for composite layers, the discrepancies between experimental results and second approach may be attributed from the selected shell elements. It can be
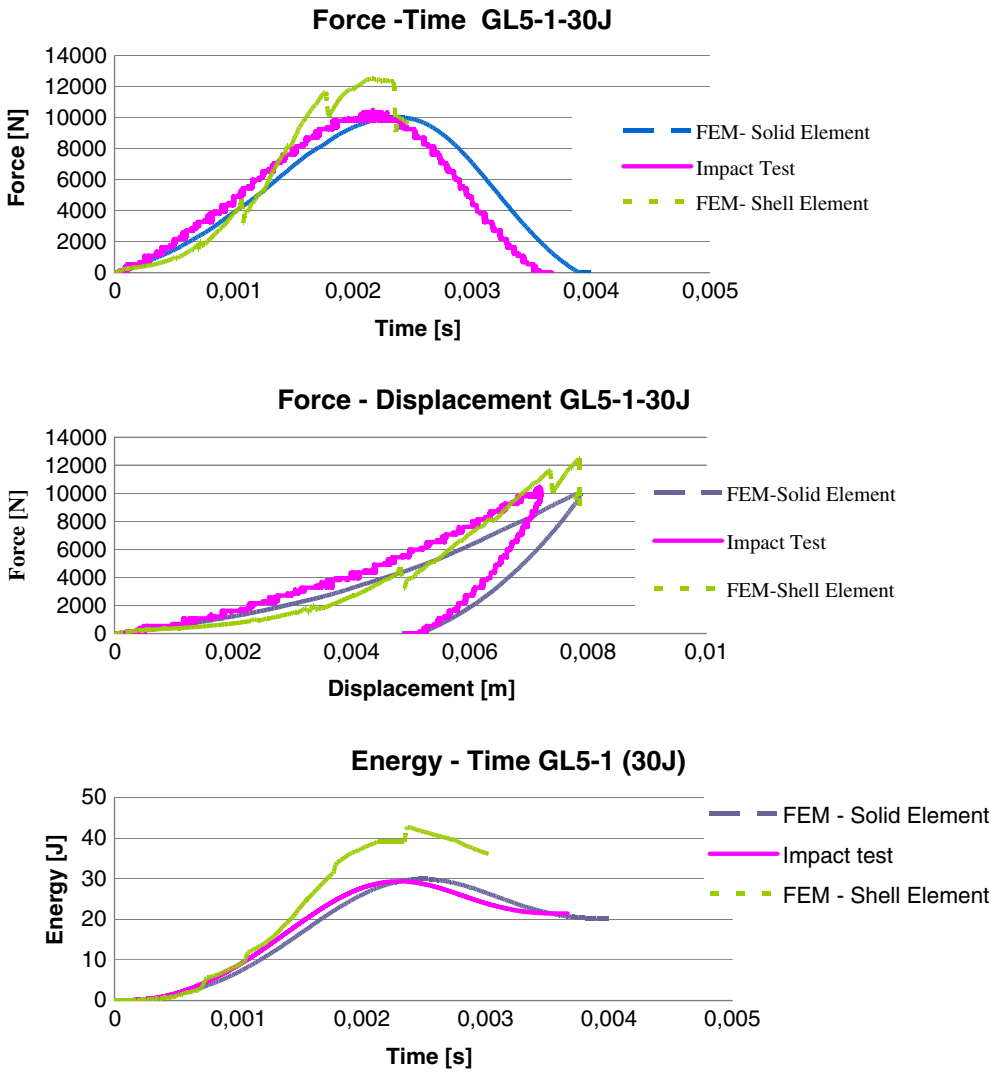

Fig. 13 Comparison of FE-predictions (energy-time) for two element types with the experimental data as reference 
concluded that in comparison to the shell element and failure criterion effects on the numerical modeling of impact resistance of FMLs, the first one has more influence on the applicability of results.

\section{Conclusions}

The present article discusses the impact resistance of FMLs in two ways: an experimental study and a numerical analysis. Two parameters have been examined relevant to the impact behavior of these materials, i.e. type of metal and its thickness within a laminate. Considering substitution aluminum by magnesium aluminum, it is shown that such replacement will not yield improvements, in spite of some apparent advantages like similar perforation energy or lower dent depth, due to considerable damage area and lower specific properties (normalized by weight of the laminate). Increasing the aluminum thickness improves the impact performance of FMLs but as it increases the weight, it should be considered where a weight penalty is acceptable.

FE modeling of FMLs under impact loading is a challenging work. Therefore, if the prediction of the overall behavior of these laminates is the main aim and no detailed failure analysis is required, some simpler approaches may be applied. The present study shows that due to the nature of impact loading and therefore the necessity of realistic modeling of the interface between the metal and fiber-reinforced composite layers, the use of continuum shell elements for the prepreg layers, (typical FE analyses), can not predict reliable results. Applying solid elements for both type of layers within FMLs, leads to a good predictions of the impact response compared to experimental results, even if incorrect failure criterion (maximum tensile stress) are assumed for composite constituent of FMLs, However, when the impact energy is too high, producing a considerable damage in the laminate, full detailed FE modeling using a suitable failure criterion applicable to solid element is required.

\section{References}

1. Vlot, A., Gunnink, J.W. (eds.): Fiber metal laminates - an introduction. Kluwer Academic Publisher, Dordrecht (2001)

2. Vlot, A.: Glare. Kluwer Academic Publisher, Dordrecht (2001)

3. Vlot, A.: Impact tests on aluminium 2024-T3, Aramid and glass reinforced laminates and thermoplastic composites. Delft Univ. of Technology, Delft (1987). Report LR-534

4. Liu, Y., Liaw, B.: Effects of constituents and lay-up configuration on drop- weight tests of fiber -metal laminates. Appl. Comp. Mater. 17, 43-62 (2010)

5. Cortes, P., Cantwell, W.J.: Fracture properties of a fiber-metal laminates based on magnesium alloy. J. Materials Science 39, 1081-1083 (2010)

6. Cortes, P., Cantwell, W.J.: The fracture properties of a fiber-metal laminate based on magnesium alloy. Composites Part B: Engineering 37, 163-170 (2006)

7. Supplit, R., Koch, T., Schubert, U.: Evaluation of the anti-corrosive effect of acid picking and sol-gel coating on magnesium AZ31 alloy. Corros. Sci. 49, 3015-3023 (2007)

8. Alderliesten, R.C., Rans, C., Benedictus, R.: The applicability of magnesium based fiber metal laminates in aerospace structures. Composite Science and Technology 68, 2983-2993 (2008)

9. Fan, J., Cantwell, W.J., Guan, Z.W.: The low -velocity impact response of fiber -metal laminates. J. Reinforced Plastics Composites, 1-10 (2010)

10. Vlot, A.: Low velocity impact loading on fiber reinforced aluminium laminates (Arall and Glare) and other aircraft sheet materials. Delft Univ. of Technology, Delft (1993). Rept. LR-718

11. Liaw, B.M., Liu, Y.X., Villars, E.A.: Impact damage mechanisms in fiber-metal laminates, Proceedings of the SEM Annual Conference on Experimental and Applied Mechanics, Portland, Oregon, June 4-6 (2001) 
12. Vlot, A.: Impact loading on fiber metal laminates. Int. J. Impact Engng 18(3), 291-307 (1996)

13. Caprino, G., Spataro, G., Del Luongo, S.: Low-velocity impact behaviour of fiberglass-aluminium laminates. Composites Part A: Applied Science Manufacturing 35, 605-616 (2004)

14. Reyes, G., Cantwell, W.J.: The mechanical properties of fiber-metal laminates based on glass fiber reinforced polypropylene. Composite Science Technology 60, 1085-1094 (2000)

15. Abdullah, M.R., Cantwell, W.J.: The impact resistance of propylene- based fiber-metal laminates. Compos. Sci. Technol. 66, 1682-1693 (2006)

16. Cortes, P., Cantwell, W.J.: The impact properties of high-temperature fiber- metal laminates. J. Composite Materials 41(5), 613-632 (2007)

17. Vlot, A., Krull, M.: Impact damage resistance of various fiber metal laminates. J. Phys IV France 7, $1045-1050$ (1997)

18. McKown, S., Cantwell, W.J., Jones, N.: Investigation of scaling effects in fiber- metal laminates. J. Composite Materials 42(9), 865-888 (2008)

19. Carrillo, J.G., Cantwell, W.J.: Scaling effects in the low velocity impact response of fiber - metal laminates. J. Reinforced Plastics Composites 27(9), 893-907 (2008)

20. Liu, Y.X., Liaw, B.M.: Drop - weight impact on fiber - metal laminates using various indenters. Proceeding of the SEM International Congress \& Exposition on Experimental and Applied Mechanics, Costa Mesa, CA, June 7-10 (2004)

21. Laliberte, J.F., Straznicky, P.V., Poon, C.: Impact damage in fiber metal laminates, Part1: Experiment. AIAA J. 43(11), 2445-2453 (2005)

22. Laliberte, J., Poon, C., Straznicky, P.V.: Numerical modeling of low-velocity impact damage in fibermetal laminates, ICAS 2002 Congress (The International Council of the Aeronautical Sciences Congress), Toronto, Canada (2002)

23. Guan, Z.W., Cantwell, W.J., Abdullah, R.: Numerical modeling of the impact response of fiber-metal laminates. Polymer Composites, 603-611 (2009)

24. Song, S.H., Byun, Y.S., Ku, T.W., Song, W.J., Kim, J., Kang, B.S.: Experimental and numerical investigation on impact performance of carbon reinforced aluminum laminates. J. Mater. Sci. Technol. 26 (4), 327-332 (2010)

25. Seo, H., Hundley, J., Hahn, H.T., Yang, J.M.: Numerical simulation of glass - fiber- reinforced aluminum laminates with diverse impact damage. AIAA J. 48(3), 676-687 (2010)

26. McCarthy, M.A., Xiao, J.R., Petrinic, N., Kamoulakos, A., Melito, V.: Modeling bird impacts on an aircraft wing - part1: material modeling of the fiber metal laminate leading edge material with continuum damage mechanics. Int. J. Crashworthiness 10(1), 41-49 (2005)

27. Hashagen, F.: Numerical analysis of failure mechanisms in fiber metal laminates, $\mathrm{PhD}$ Thesis, Delft University of Technology (1998)

28. Linde, P., Pleitner, J., de Boer, H., Carmone, C.: Modeling and simulation of fiber metal laminates, ABAQUS Users' Conference, Boston, Massachusetts (2004)

29. Hagenbeek, M.: Characterisation of fibre metal laminates under thermomechanical loadings, $\mathrm{PhD}$ Dissertation, TUDelft, The Netherlands (2005)

30. Agnew, S.R., Duygulu, O.: Plastic anisotropy and the role of non-basal slip in magnesium alloy AZ31B. Int. J. Plasticity 21, 1161-1193 (2005) 